Revista de Derecho

\title{
Panorama actual del bitcóin. Una descripción práctica y jurídica de las criptomonedas en Colombia y Ecuador
}

\section{Current Panorama of Bitcoin. A practical and Legal Description of Cryptocurrencies in Colombia and Ecuador}

Franco Daniel Valencia Marín

Investigador independiente

Lille, Francia

francodaniel.valenciamarin.etu@univ-lille.fr

ORCID: 0000-0002-8103-5971

Artículo de investigación

DOI: https://doi.org/10.32719/26312484.2021.36.3

Fecha de recepción: 31 de diciembre de 2020

Fecha de revisión: 20 de enero de 2021

Fecha de aceptación: 16 de marzo de 2021

Fecha de publicación: 1 de julio de 2021 


\section{RESUMEN}

El bitcóin es una tecnología originada en el año 2009 que pretende implementar un sistema de pagos descentralizado entre personas. El presente trabajo se realiza con el fin de dar a conocer dicha tecnología, la cual denota importancia debido a las funcionalidades y oportunidades que ofrece para todo tipo de individuos, desde aquellos que buscan un refugio de valor para su patrimonio, hasta los que desean invertir su dinero o simplemente entender su funcionamiento y utilizarla en el día a día.

Este escrito expone los orígenes del bitcóin, así como sus principales características y funcionamiento. Además, analiza sus ventajas y desventajas desde un punto de vista práctico y hace un énfasis especial en la regulación jurídica existente alrededor de las criptomonedas en Colombia y Ecuador.

A través de una metodología descriptiva y utilizando herramientas cualitativas y cuantitativas, se logró determinar un crecimiento progresivo del bitcóin durante los últimos años, no solo en términos de valor, sino también de adopción y de seguridad. Por este motivo, tanto Colombia como Ecuador deben adaptar sus regulaciones jurídicas, con el fin de estar a la altura de la era digital en la que nos encontramos y aprovechar con ello los beneficios de este fenómeno en lugar de impedir su desarrollo.

PALABRAS ClAVE: bitcóin, criptomonedas, blockchain, descentralización, regulación, Colombia, Ecuador, criptoactivos.

\section{ABSTRACT}

Bitcoin is a technology originated in 2009 that aims to implement a decentralized payment system between people. The present work is carried out in order to publicize this technology, which denotes importance due to the functionalities and opportunities it offers for all types of individuals, from those who seek a refuge of value for their assets, to those who wish to invest your money or simply understand how it works and use it on a day-to-day basis.

This writing exposes the origins of Bitcoin, as well as its main characteristics and operation. In addition, it analyzes its advantages and disadvantages from a practical point of view and places special emphasis on the existing legal regulation around cryptocurrencies in Colombia and Ecuador.

Through a descriptive methodology and using qualitative and quantitative tools, it was possible to determine a progressive growth of Bitcoin in recent years, not only in terms of value, but also of adoption and security. For this reason, both Colombia and Ecuador must adapt their legal regulations, in order to live up to the digital age in which we find ourselves and thereby take advantage of the benefits of this phenomenon instead of preventing its development.

KEYWORDS: Bitcoin, crypto-currencies, blockchain, decentralization, regulation, Colombia, Ecuador, crypto-assets. 


\section{INTRODUCCIÓN}

El dólar es el dinero del gobierno, el oro y la plata son las monedas de Dios y el bitcóin es el dinero de la gente. Robert Kiyosaki. ${ }^{1}$

$\mathrm{E}$ n el último siglo varios sectores de la economía y de la sociedad han experimentado un cambio radical. En un primer momento gracias a la industrialización y recientemente, gracias a la tecnología. Algunos ejemplos son los sectores de la salud, la construcción, el transporte y la administración pública, los cuales han evolucionado para adaptarse a la época digital.

Sin embargo, más allá de las exigencias propias de la digitalización, uno de los que no ha cambiado a grandes rasgos es el sector financiero, más cuando se le compara con otros como los mencionados. Los bancos no han conocido un cambio importante desde el momento de su aparición y consolidación en la edad media. ${ }^{2}$ Su naturaleza ha sido siempre la administración del dinero, brindando un servicio en contrapartida, con el fin de obtener rendimientos, los cuales en la mayoría de casos benefician principalmente al banco y no a las personas que proporcionan el capital.

Este sistema exclusivamente centralizado y que es calificado por algunos como injusto, está siendo amenazado por una tecnología revolucionaria que hizo su entrada en el año 2009, las criptomonedas, siendo el bitcóin la primera y principal. "Bitcóin es una red consensuada que permite un nuevo sistema de pago y una moneda completamente digital. Es la primera red entre pares de pago descentralizado impulsado por sus usuarios sin una autoridad central o intermediarios". ${ }^{3}$

Contrario a las monedas fiduciarias, como el dólar o el peso, no existe autoridad central que se encargue de crear los bitcóins y que ostente su control. Tal entidad es, en este ecosistema, remplazada por millones de ordenadores repartidos a través del planeta, conocidos como nodos. Estos se encargan, entre otras cosas, de verificar cada transacción realizada y de registrarla en la "Blockchain", una especie de libro contable abierto y accesible a todos que garantiza la transparencia, seguridad y fortaleza de la red.

1. Robert Kiyosaki, FALSO: Dinero falso, falsos maestros, falsos activos: cómo las mentiras están haciendo a los pobres y a la clase media más pobres, 2019, 40.

2. "Historia de los bancos", Comisión para el Mercado Financiero CMFEduca, accedido 30 de diciembre de 2020, https://bit.ly/34YweUu.

3. “Qué es Bitcoin?”, Bitcoin.org, accedido 30 de diciembre de 2020, http://bit.ly/384FRDo. 
El presente trabajo se focaliza principalmente en el bitcóin como medio de pago descentralizado y digital entre personas. En él se evocarán de manera detallada sus orígenes, sus objetivos y su funcionalidad, así como sus ventajas e inconvenientes, haciendo énfasis en el impacto que este representa en la sociedad, así como su regulación jurídica en Colombia y Ecuador.

El análisis realizado permitirá al lector determinar con base en la información proporcionada y en sus propias convicciones, si el bitcóin constituye un activo de inversión, un refugio de valor, un fenómeno capaz de remplazar el sistema financiero tradicional o si, por el contrario, se trata de la simple especulación.

\section{EL BITCÓIN COMO REVOLUCIÓN DIGITAL DEL SISTEMA MONETARIO}

El ser humano ha utilizado diferentes medios para intercambiar valor, los cuales fueron reemplazados progresivamente por objetos que lo representaran mejor y que aseguraran cada vez más su "vendibilidad" en el espacio y el tiempo, como lo menciona Saifedean AMMOUS, en su libro El Patrón Bitcoin. ${ }^{4}$

Dichos objetos han sido diferentes a lo largo de la historia, yendo desde conchas de mar, piedras y metales preciosos, hasta las monedas y billetes que conocemos en la actualidad y que son emitidos por una autoridad central, conocidos como dinero fiduciario. ${ }^{5}$ Es en este hilo económico de la historia hace su aparición el bitcóin como una alternativa al sistema financiero tradicional, el cual, como muchos otros que han existido anteriormente, posee ciertas ventajas e inconvenientes.

\section{ORÍGENES DEL BITCÓIN:}

\section{CARACTERÍSTICAS, FUNCIONAMIENTO Y OBJETIVOS}

El bitcóin, como otros criptoactivos, encuentra su origen en la criptografía, disciplina "utilizada para salvaguardar los datos e impedir que terceros no autorizados puedan acceder a información valiosa o alterarla para su propio beneficio o en perjuicio de otros". ${ }^{6}$ Dentro de la criptografía existe una técnica conocida como cifrado, la cual

\footnotetext{
4. Saifedean Ammous, El Patrón Bitcoin. La alternativa descentralizada a los bancos centrales (Barcelona: Deusto Grupo Planeta, 2018), 36.

5. "Historia del dinero: del trueque a las criptomonedas", Blog Bankia, 26 de abril de 2016, http://bit. 1y/2XbWy9L.

6. “¿Qué es la criptografía?”, Bit2Me Academy, accedido 30 de diciembre de 2020, https://bit.ly/382i2vT.
} 
pretende asegurar la identidad del autor de una comunicación, de volver ilegible una información a personas no autorizadas y de garantizar con ello su seguridad. ${ }^{7}$

Antes de 1970, el cifrado se utilizaba principalmente con fines militares y diplomáticos y era monopolizado por el Estado. En esta época, la era digital aún no había llegado, ya que cuando lo hizo, la criptografía se democratizó. ${ }^{8}$ Detrás de este movimiento libertario apareció un grupo de personas expertas en dicha área, conocidas como "Cypherpunks", los cuales tenían como objetivo defender el derecho a la privacidad en internet a través del uso de herramientas tecnológicas. Su manifiesto publicado en 1993, que puede encontrarse en el sitio web Activism.net, es un claro ejemplo de sus ideologías.

Nosotros, los Cypherpunk, estamos dedicados a la construcción de sistemas anónimos. Defendemos nuestra privacidad a través de la criptografía, los sistemas de mensajería anónima, las firmas digitales y el dinero electrónico.

El primer paso hacia las monedas digitales se remonta a los años 80, cuando David Chaum, celebre criptógrafo y matemático americano, comenzó a escribir sobre la moneda digital anónima y los sistemas de reputación seudónimos. Posteriormente, en los años 90, proyectos precursores del bitcóin hicieron su entrada, Hashcash, B-Money y Bit Gold, etc. Todos compartían elementos que hoy en día se encuentran presentes en la tecnología de bitcóin y de otras criptomonedas.

En 2008 Satoshi Nakamoto publicó el libro blanco de bitcóin, en el cual cita y se apoya en varios proyectos y criptógrafos enunciados. Las ideas contenidas en este documento son una muestra clara de la afinidad de Satoshi con el movimiento Cypherpunk, sobre todo en lo que concierne la protección de la privacidad y la posibilidad para los individuos de controlar su propio dinero.

En el White Paper, Nakamoto precisó:

El modelo bancario tradicional logra un nivel de privacidad al limitar el acceso a la información de las partes envueltas y del tercero confiado. La necesidad de anunciar todas las transacciones públicamente se opone a este método, pero la privacidad aún puede ser mantenida al romper el flujo de la información en otro lugar: al mantener las claves públicas anónimas. El público puede ver que alguien está enviando una cantidad a otra persona, pero sin información que relacione la transacción a ninguna persona. ${ }^{9}$

7. “¿Qué es el cifrado de datos?”, Kaperski, 26 de abril de 2016, http://bit.ly/380cm5m.

8. "Le mouvement Cypherpunk", Horizen Academy, accedido 30 de diciembre de 2020, http://bit. ly/3aXTndS.

9. "Bitcoin: Un sistema de pago electrónico persona a persona", Bitcoin.org, 2009, http://bit.ly/2WXNNQv. 
Nakamoto consideró en su proyecto un sistema compuesto por seis elementos principales. En primer lugar, el bitcóin es antes que nada un protocolo abierto que establece un sistema de pago entre personas, a través de la creación de una moneda electrónica, los bitcóins (con b minúscula o BTC). Es caracterizado por su practicidad y accesibilidad, ya que cualquier persona con conexión a internet y sin requerir grandes conocimientos técnicos puede utilizarlo.

En segundo lugar, la seguridad y el control del dinero son principios fundamentales. Las transacciones en la red de bitcóin están protegidas por un hash criptográfico, algoritmo que se encarga de cifrar los datos, protegiéndolos a través del uso de claves privadas, que tienen por objetivo verificar que cada transacción sea correcta.

De igual forma, la característica de disponibilidad está presente, puesto que bitcóin funciona en todos los lugares donde exista conexión a internet y en cualquier momento. Es suficiente tener un portafolio electrónico o "wallet" para guardar los bitcóins, enviarlos y/o recibirlos. De esta manera bitcóin puede mejorar notablemente algunas operaciones financieras tradicionales, haciéndolas más económicas y rápidas.

Aunado a lo anterior, la privacidad de la red es fundamental. Con el bitcóin no existe un número de tarjeta bancaria, ni informaciones personales asociadas a las billeteras, incluso existen otras criptomonedas que, privilegiando la confidencialidad, fueron creadas para aumentar aún más el anonimato, como es el caso de Monero y Zcash.

Por último, bitcóin no está limitado a la simplicidad de un sistema de pago. La cantidad de bitcóins que existirán está definida por el propio código. Solo serán creadas 21 millones de monedas y según el explorador de bloques del sitio Blockchain.com, al 26 de noviembre de 2020, un total de 18,5 millones de BTC habían sido creados, cantidad que aumenta diariamente. Estas características le proporcionan a la moneda un carácter deflacionista, contrario al carácter inflacionista, propio del sistema monetario tradicional.

Habiendo descrito los orígenes de bitcóin, así como sus principales características, es necesario profundizar sobre tres nociones esenciales para comprender correctamente su funcionamiento.

\section{La tecnología de cadena de bloques o "Blockchain"}

La Blockchain puede ser definida como un gran libro contable abierto al público. ${ }^{10}$ Se trata de una tecnología de registro y transmisión de información que funciona sin

10. “Blockchain: Qué es, cómo funciona y cómo se está usando en el mercado", WeLiveSecurity by Eset, 4 de septiembre de 2018, http://bit.ly/3pBCJo9. 
un órgano de control. Técnicamente, es una base de datos distribuida, en la cual las informaciones enviadas por los participantes y las demás informaciones vinculadas son verificadas y agrupadas en intervalos de tiempo regulares conocidos como "bloques", los cuales se unen a través del tiempo para formar así una cadena.

En el caso de bitcóin, cada transacción se agrupa a otras para formar bloques de datos, que contienen una gran cantidad de transacciones, los cuales, después de una validación, son incorporados a la cadena de manera definitiva. Todas las transacciones realizadas en la red de bitcóin son públicas y accesibles en la Blockchain. Una transacción de BTC contiene la dirección bitcóin del emisor, la cual debe tener los fondos para el pago, la dirección del destinatario y la cantidad de bitcóins que serán transferidos.

\section{Un algoritmo criptográfico que utiliza la modalidad de cifrado asimétrico}

Las llaves de cifrado son utilizadas para autorizar, asegurar y verificar las transacciones. Dado que el cifrado utilizado es asimétrico, el sistema de bitcóin utiliza un par de claves, cada una compuesta de una larga serie de cifras. Una llave es pública y controla la operación de descifrado, mientras que la otra es privada y se encarga de la operación de cifrado o viceversa. Para el algoritmo, no hay dificultad en crear una clave privada y una pública; sin embargo, determinar una clave privada a partir de la clave pública correspondiente no es posible desde un punto de vista computacional. Esto permite a los usuarios de la red compartir tranquilamente su clave pública con el fin de enviar y recibir bitcóins, a los cuales pueden acceder y disponer de ellos libremente gracias a su clave privada.

De igual forma, un emisor firma digitalmente una transacción de bitcóin con su clave privada y en esta transacción esta automáticamente incluida la clave pública. Utilizando esta última, el sistema verifica que la firma digital es válida y, por tanto, confirma que se trata del emisor correcto, así: 


\section{Figura 1. Ejemplo de una transacción de bitcóin}
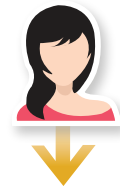

Alicia paga 250 BTC

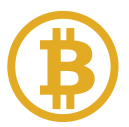

Alicia genera una transacción de Bitcoins personal a Bob.
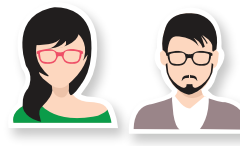

Otras personas pueden verificar que Alicia ha enviado su transacción correctamente

La transacción se envía a los nodos de Blockchain. Estos nodos comprueban las transacciones, comparando los hash de los mensajes con los de las firmas y los envían a sus nodos sucesivos.

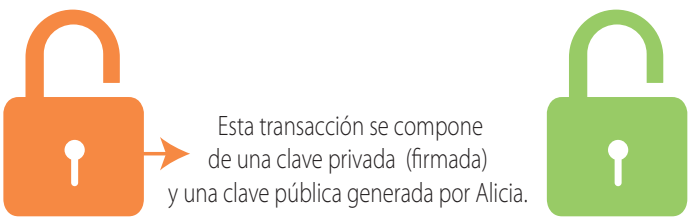

Clave privada de Alicia khfpoojnb jhj.......
Clave pública de Alicia

puwy690PPs

Se genera un código hash y se firma con la clave privada, se le añade la clave pública para comprobar la firma y el monedero de Alicia suelta la transacción a la red de Bitcoin para que sea procesada.

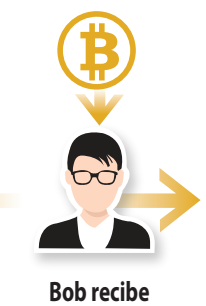

el pago de 250 BTC
Bob recibe la transacción, efectúa un hash del mensaje y desencripta la firma con la clave pública, compara el hash del mensaje con el de la firma para ver que coincidan.

Fuente y elaboración: Openclassrooms, curso: "Comprendre le Bitcoin et la Blockchain"."

\section{Una red de nodos, conocidos como "mineros"}

La descentralización de bitcóin reposa en un conjunto de nodos que participan de manera voluntaria en el sistema y que están distribuidos por todo el planeta. Son conocidos como "mineros", ya que participan tanto en la validación de los bloques de transacciones, como en el funcionamiento y actualización de la Blockchain. Además, son ellos quienes con su actividad crean los nuevos bitcóins a través de una operación conocida como "minería".

11. “Comprendre le Bitcoin et la Blockchain”, Openclassrooms, 28 de diciembre de 2017, http://bit.ly/3pHZ2c7. 
El software de bitcóin que los mineros utilizan posee el protocolo de bitcóin con el conjunto de reglas y configuraciones necesarias para su funcionamiento. La Blockchain de bitcóin debe actualizarse constantemente y cada 10 minutos aproximadamente, un nuevo bloque es añadido a la cadena con las últimas transacciones validadas. Son los mineros quienes realizan dicha tarea, quienes pueden ser personas naturales o jurídicas. Actualmente la mayor parte del poder de minado de la red se concentra en industrias ubicadas estratégicamente. Todos los mineros utilizan componentes informáticos diseñados exclusivamente para esta tarea, con la finalidad de generar ingresos y de contribuir con el desarrollo de la red.

Los mineros trabajan para validar y adjuntar el próximo bloque a la cadena. Sin embargo, solo un bloque se añadirá. El minero que encuentre en primer lugar la solución al algoritmo que exige el protocolo de bitcóin, será el ganador de las comisiones percibidas en cada transacción del bloque, así como la recompensa por haberlo minado, la cual corresponde a una cantidad de bitcóins. Actualmente, esta asciende a 6,25 BTC. El protocolo prevé una disminución a la mitad de esta recompensa cada 4 años, fenómeno que se conoce como "halving". Al inicio de bitcóin, la recompensa era de 50 bitcóins y ha ido y seguirá disminuyendo progresivamente.

Finalmente, una vez el algoritmo ha sido solucionado, el resto de mineros reciben el bloque y su solución con el fin de autentificarlo y validarlo, es decir, el resto de la red de nodos confirman que la solución encontrada por el primer minero es correcta. Si es el caso, el bloque será añadido a la Blockchain y quedará registrado para siempre. Esta operación se repite consecutivamente.

Una vez desarrollados los conceptos clave del bitcóin, es necesario profundizar en los aspectos positivos y negativos que esta tecnología representa en nuestra sociedad, con el fin de conocer las funcionalidades prácticas de este fenómeno. 


\section{Figura 2. Proceso de minería de una transacción de bitcóin}

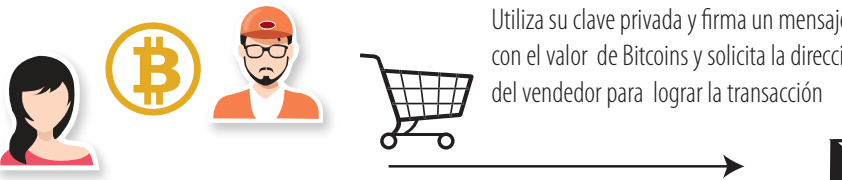

Alicia quiere comprar productos usando Bitcoins

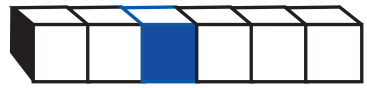

La transacción solicitada por Alicia está agrupada en un bloque con otras transacciones

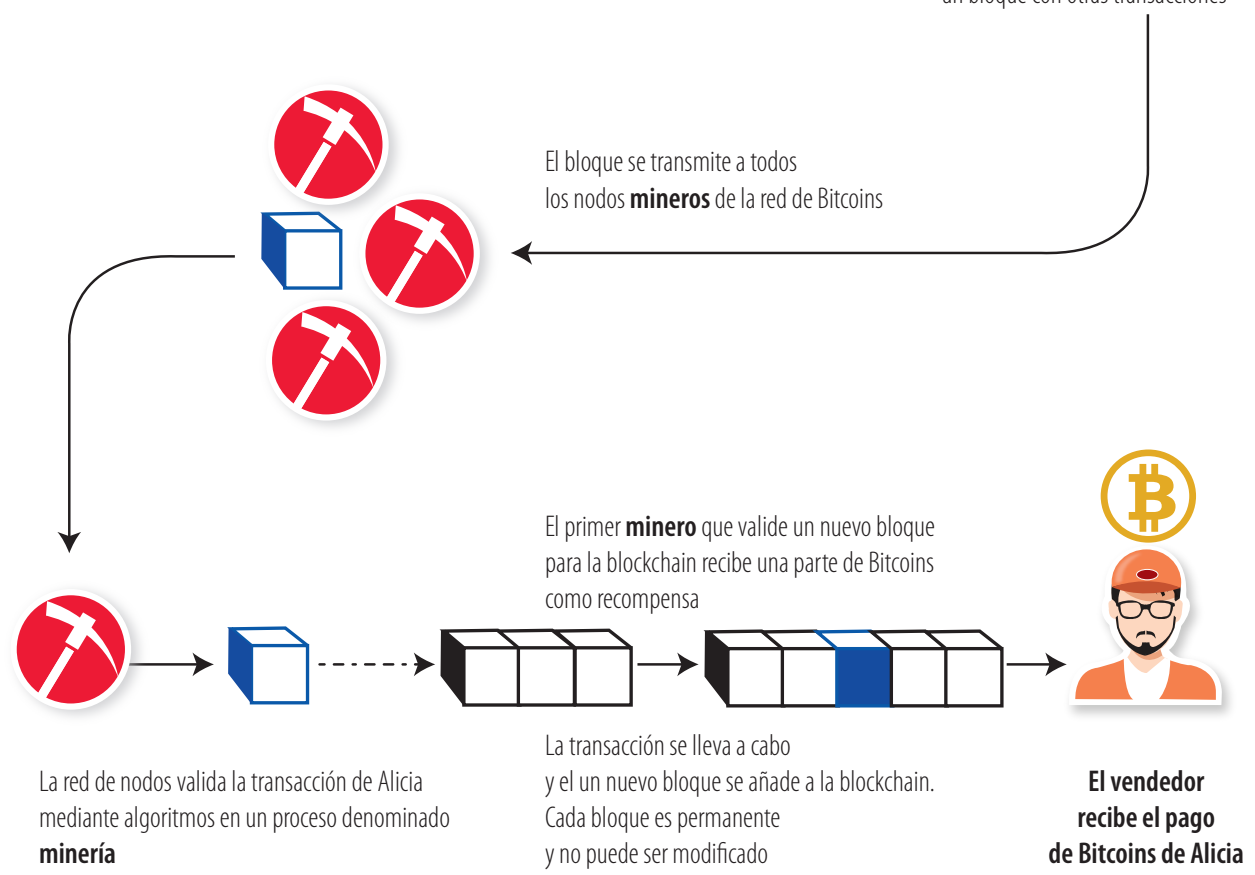

Fuente y elaboración: Bitpanda Academy, "En quoi consiste le 'mining de bitcoins" ".12

\section{LAS VENTAJAS E INCONVENIENTES DE BITCÓIN}

Tanto los puntos positivos como negativos de bitcóin han cambiado a través de los años, puesto que los inconvenientes del sistema en su época embrionaria no son los

12. "En quoi consiste le 'mining de bitcoins "”, Bitpanda Academy, accedido 26 de diciembre de 2020, http:// bit.ly/3rHOQ55. 
mismos que en la actualidad. A lo largo de los años, el bitcóin se ha vuelto cada vez más fuerte, dejando de lado sus principales debilidades, para abrirse un lugar como un activo importante, dirigido a un público más amplio.

\section{El rol de bitcóin como refugio de valor}

Las divisas tradicionales reúnen ciertas condiciones para ser consideras como monedas legales y así cumplir las funciones para las que fueron creadas. Las características que deben tener son: aceptación universal, facilidad de transporte y almacenamiento, divisibilidad e incorruptibilidad. Estas características son indispensables para que el dinero cumpla sus funciones y sirva como medio de intercambio y de pago, como unidad de cuenta y reserva de valor.

En el caso de bitcóin, este cumple con la mayoría de características y funciones para ser considerado como dinero. En efecto, los bitcóins son digitales y, por tanto, fáciles de transportar y almacenar. Además, pueden ser divididos fácilmente, cada uno equivale a 100'000.000 de "satoshis". Por último, en términos de incorruptibilidad, gracias a su código y a la tecnología Blockchain, los bitcóins no pueden ser falsificados y su red está diseñada para ser protegida frente a ciberataques.

Sin embargo, el bitcóin no es reconocido como una moneda legal, ya que no está respaldada por ningún gobierno u organización y, por tanto, no posee aceptación universal, además su gran volatilidad impide que sea considerado como reserva de valor. No obstante, aunque es cierto que el precio del bitcóin ha sufrido una volatilidad enorme a lo largo de su historia, durante el año 2020 este ha proporcionado rendimientos más significativos que otros vehículos de inversión, como el oro o las principales acciones, como el índice S\&P 500 de Estados Unidos. ${ }^{13}$ Además, debido a su carácter deflacionista, este no se ve afectado por la inflación que caracteriza las divisas gubernamentales, las cuales en el transcurso de los últimos años han perdido progresivamente su valor, ${ }^{14}$ situación que pone en duda la incapacidad de bitcóin para cumplir esta función.

Sin embargo, economías en crisis, como Venezuela, Argentina o el Líbano, donde la inflación ha sobrepasado limites exorbitantes, son algunos países donde bitcóin puede servir como refugio de valor. En Venezuela, por ejemplo, según un reporte

13. Eseandre Mordi, "Bitcoin Is Twice as Profitable as Gold and the S\&P500 Index in 2020", TheTradable. com, 1 de octubre de 2020, http://bit.ly/3b16BX5.

14. “U.S. Dollar Index.43 Year Historical Chart", Macrotrends.net, accedido 30 de diciembre de 2020, http:// bit.ly/2L6uUIq. 
de la Asamblea Nacional, ${ }^{15}$ entre enero y septiembre de 2020 la inflación fue de un $1433,58 \%$. Como consecuencia de esta situación, muchas personas han decidido transformar su patrimonio en este criptoactivo. Precisamente, un estudio reciente de la plataforma Coin Dance ${ }^{16}$ muestra que el país con mayor actividad de compra y venta de criptomonedas en Latinoamérica es justamente Venezuela, con el $51 \%$ de operaciones, seguido por Colombia con un $25 \%$.

En el caso de Argentina y el Líbano, sus monedas presentan también graves dificultades. Para el país suramericano, entre 2002 y 2019, la inflación acumulada fue de un $1169,4 \%$, lo que significa un promedio anual del $20,43 \%{ }^{17}$ y en el caso del Líbano, este vive la peor crisis económica desde 1975, cuando se encontraba en una guerra civil. $^{18}$

Dichas cifras son aún más impresionantes cuando las comparamos con el crecimiento del precio del bitcóin, el cual solo durante el año 2020, se ha valorizado más de un $278 \%$, desde el 1 de enero hasta el 28 de diciembre, pasando de $\$ 7300$ a un máximo de $\$ 28.000$ por unidad.

\section{Las transferencias de fondos internacionales}

El Banco Mundial estima que: "Los flujos anuales de remesas a países de ingreso bajo y de ingreso mediano registradas oficialmente alcanzaron los USD $529.000 \mathrm{mi}$ llones en 2018, lo que representa un aumento del 9,6\% con respecto al récord anterior, de USD 483.000 millones en 2017". ${ }^{19}$

Esta actividad conlleva una carga pesada para los usuarios del sistema bancario o de las casas de cambio, respecto a las comisiones que estos cobran por participar en la cadena de transacción, lo cual vuelve poco prácticas las transferencias de dinero hacia otros países, realizadas principalmente por migrantes que envían fondos para el sostenimiento de sus familias.

15. "La inflación en Venezuela fue del 1433,58\% hasta septiembre, según el Parlamento", Agencia EFE, 8 de octubre de 2020, http://bit.ly/3htG8mm.

16. “¿Cuáles son los 6 países que más comerciaron con bitcoin en Latinoamérica?”, Cripto 247, 16 de junio de 2020, https://bit.ly/394EVyb.

17. Federico Caballero, "La inflación en Argentina: una explicación monetaria", Economipedia Blog, 14 de septiembre de 2020, http://bit.ly/3aRthJo.

18. Chloè Domat, "Sa monnaie ne vaut plus rien, le Liban plonge dans l'abîme", Ouest France presse, 11 de julio de 2020, https://bit.ly/3n0z0z2.

19. "Cifra sin precedente de remesas a nivel mundial en 2018", Banco Mundial, abril 8 de 2019, http://bit. ly/2JB2cio. 
Con la tecnología de bitcóin, este problema se resuelve considerablemente. En efecto, los usuarios pueden disminuir el costo de una transacción internacional y también el tiempo de envío a un promedio de 10 minutos. Por ejemplo, para un envío de $\$ 1000$ en BTC a cualquier lugar del mundo, el emisor debe pagar una tasa de transacción aproximada de 0,0005 satoshis, equivalente a $\$ 13$ al precio actual de BTC, según informaciones del sitio web Coingecko, lo que corresponde solamente a un 1,3\%, suma bastante inferior a lo que cobran los bancos y casas de cambio.

Este dinero llega en bitcóin al portafolio del destinatario, quien tiene la posibilidad de vender los bitcóins a su moneda local o utilizarlos directamente para adquirir bienes y servicios. Por lo tanto, el bitcóin y otros criptoactivos mejoran significativamente el mercado de transferencias internacionales e incluso nacionales, a través de un sistema más rápido, autónomo y económico. Algunas otras criptomonedas enfocadas específicamente en este servicio, son Litecoin (LTC) y Stellar (XLM), las cuales prometen tiempos de entrega mucho más rápidos y transferencias más simples.

No obstante, existen inconvenientes que deben ser superados con el fin de que el sistema funcione correctamente. En primer lugar, bitcóin aún no es suficientemente utilizado y respaldado en la sociedad. La mayoría de personas no tienen conocimiento sobre esta tecnología y esto impide su uso y expansión. Además, la imposibilidad de usar el bitcóin y otras criptomonedas para comprar bienes o servicios directamente desmotiva fuertemente a los usuarios. Por último, es indispensable tener una conexión a internet y un portafolio electrónico para acceder a la red de bitcóin. Actualmente, hay poblaciones que no tienen acceso a internet, sobre todo en regiones de África y de América Latina, lugares donde las remesas son bastante frecuentes debido al gran número de migrantes provenientes de estos territorios. Esta situación, sumada a la poca educación en temas informáticos, impide la adopción masiva de esta tecnología.

\section{La seguridad de la red}

La seguridad del protocolo de bitcóin es directamente proporcional a la fortaleza de su red. Los nodos que se encargan de validar cada bloque de transacciones y de actualizar la Blockchain son el corazón del sistema. En términos de adopción, hay una gran cantidad de nuevos usuarios que usan los bitcóins por diferentes motivos, ya sea de inversión, especulación o como reemplazo al dinero fiduciario. De igual forma, 
nuevos mineros han entrado en el protocolo, grandes empresas que ponen a disposición de la red miles de máquinas concebidas específicamente para esta tarea. ${ }^{20}$

Estas empresas se asocian en grupos de minería y han industrializado esta actividad, lo que permite que la red sea cada vez más grande, fuerte y evolucione constantemente. Además, la entrada progresiva de actores institucionales en el ecosistema ha producido que el precio de bitcóin aumente parabólicamente, así como la confianza, popularidad y utilidad del activo digital.

Sin embargo, paradójicamente el crecimiento de la red y sobre todo la centralización de los pools de minería, es al mismo tiempo un peligro para el bitcóin. Según una estadística reciente, China controla el $65 \%$ del poder de minería de la red. ${ }^{21}$ Esto significa que la mayoría de mineros se encuentran en este territorio, lo que representa en la tecnología Blockchain, una amenaza potencial denominada como "ataque del $51 \%$ ", 22 el cual consiste en un común acuerdo entre los mineros de la red, quienes llegan a controlar más del $51 \%$ del poder de cálculo que administra la cadena.

Normalmente, si un solo minero introduce intencionalmente un error o intenta piratear la cadena, todos los demás mineros automáticamente anularían dicho ataque y corregirían prontamente la incidencia. Sin embargo, si una cooperativa reagrupa más del $50 \%$ de la capacidad de minería, sería posible alterar y controlar la Blockchain.

Este tipo de ataque tendría por objetivo destruir la confianza de los usuarios en una cadena de bloques, desmotivando así su utilización. Además, en el caso particular de bitcóin, esto haría caer el valor del criptoactivo si el atacante decidiera bloquear las transacciones $\mathrm{u}$ otorgarse unidades de la criptomoneda. No solo es el bitcóin quien sería susceptible de sufrir este tipo de evento, sino también otros criptoactivos que estén basados en la misma tecnología, como reportó el 29 de diciembre de 2020 el periódico online DiarioBitcoin en su sitio web, sobre el ciberataque realizado al protocolo Cover. ${ }^{23}$

Una brecha de seguridad de este tipo no tendría vocación a presentarse en estos mismos términos en el sistema financiero tradicional. Aunque existen otros riesgos, el bitcóin es considerado mucho más peligroso que las divisas gubernamentales y, en consecuencia, que las inversiones realizadas en los mercados financieros tradicionales.

20. Andrea Leal, “2021 promete mayor expansión de granjas mineras de Bitcoin en Estados Unidos y Canadá”, CriptoNoticias, 24 de diciembre de 2020, http://bit.ly/394K2OT.

21. Florian Bayard, "Le Bitcoin (BTC) est-il trop dépendant de la Chine?”, Cryptoast, 29 de septiembre de 2020, http://bit.ly/38NnqSE.

22. Charles McFarland et al., "Informe sobre amenazas contra Blockchain", McAfee, accedido 30 de diciembre de 2020, https://bit.ly/2JyVHN3.

23. Angel Di Matteo, "Proyecto DeFi Cover Protocol fue víctima de un hackeo, pero los atacantes devolvieron los fondos", DiarioBitcoin, 29 de diciembre de 2020, https://bit.ly/2LbYD21. 
Además, al ser un sistema centralizado, en el caso de crisis o inconvenientes particulares que involucren los fondos de los usuarios, este posee dispositivos de protección para cubrir estas eventualidades, lo cual no sucede en el caso de los criptoactivos. No obstante, este panorama parece cambiar poco a poco, ya que actores privados intentan acercar cada vez más el sector de los seguros tradicionales a las criptomonedas. ${ }^{24}$

\section{La ciberdelincuencia y la multiplicidad de estafas}

Una de las críticas más fuertes hacia las criptomonedas, es la capacidad para los criminales de utilizarlas para actividades ilícitas, como el blanqueo de capitales, la compra de armas, drogas y otros bienes y servicios ilegales en los mercados de la darkweb. En efecto, el carácter digital de la moneda, la confidencialidad que la red ofrece, la falta de regulación alrededor de los criptoactivos, así como su rapidez y facilidad de transferencia, son elementos perfectos para alguien que desee utilizarlo para estos fines.

Sin embargo, un artículo de investigación reciente del equipo periodístico de Buzfeednews, publicado en su sitio web, muestra que no son los criptoactivos los más utilizados en actividades criminales, al contrario, son los bancos tradicionales los vehículos principales para ello. El estudio muestra que los bancos prestan sus servicios a delincuentes para blanquear el dinero y transferir grandes sumas provenientes de actividades ilícitas, sumas que en el estado actual del mercado cripto, no es posible comercializar fácilmente debido a la poca liquidez que este tiene, en comparación con otros.

Además, en el caso particular de bitcóin, algunas compañías han desarrollado soluciones informáticas que permiten analizar en detalle la Blockchain y las transacciones ejecutadas, permitiendo incluso identificar a la persona que se encuentra al origen de los movimientos, situación que se suma a los deberes impuestos a las plataformas de intercambio de criptomonedas, quienes están obligadas a recolectar datos personales de los usuarios que operen dentro de estas, lo que vuelve menos atractiva la moneda para cometer ilícitos.

De igual forma, es necesario precisar que la mayoría de estafas y fraudes que existen en el sistema financiero tradicional, han sido simplemente transpuestas a bitcóin y al ecosistema de criptomonedas de una forma general. Por ejemplo, una gran canti-

24. "Pólizas de seguro para las criptomonedas, un nuevo mercado en auge", INESE, 28 de julio 2020, http:// bit.ly/2L3Fks9. 
dad de personas han sido estafadas a través de empresas criminales conocidas como "scams", que no son más que fraudes de tipo piramidal.

Por último, al tratarse de dinero electrónico, los usuarios también pueden verse afectados por otros ciberataques, como aquellos basados en la ingeniería social, el phishing o el malware. Como ejemplo de estas amenazas, se observa el ciberataque sufrido por Twitter en julio de $2020^{25}$ y la reciente fuga de datos del fabricante de carteras digitales, Ledger. ${ }^{26}$

Por este motivo, gobiernos como el de Colombia ${ }^{27}$ resaltan que las denuncias impuestas que resultaren de estafas ligadas a criptomonedas no cuentan con respaldo estatal, convirtiendo esto en una gran desventaja para los usuarios de esta tecnología y sin duda un factor a tener en cuenta antes de involucrarse en este mundo.

En este orden de ideas, habiendo analizado desde una perspectiva dual los beneficios y las dificultades del bitcóin y de las criptomonedas en general, es necesario sumergirse en un aspecto crucial, la regulación jurídica existente alrededor de este fenómeno. Al respecto, debe decirse que, tratándose de un tema particularmente reciente, no existe un cuadro jurídico integral en ningún país de Latinoamérica. Colombia, por su parte, se encuentra en proceso de regulación, mientras que Ecuador se ha mostrado abiertamente en contra de las criptomonedas.

\section{UNA REGULACIÓN JURÍDICA VAGA PERO PROGRESIVA}

Hace 5 años el bitcóin y otros criptoactivos circulaban y se utilizaban sin ninguna reglamentación. Sin embargo, en 2017 el BTC alcanzó su máximo histórico, \$20.000 por unidad. Este hecho llamó la atención no solo de las personas, sino también de diferentes gobiernos y entidades reguladoras, quienes comenzaron a analizar este fenómeno para emitir conceptos y regularlo progresivamente.

Hoy, 4 años después, varias regulaciones existen, algunas, como en el caso de Colombia (A) y Ecuador (B) todavía no son integrales y no abordan ampliamente el tema, a diferencia de algunos países de la Unión Europea, como es el caso de Francia,

25. “Twitter hack: Exchange blocked 1,000 Bitcoin transactions", BBC News, 21 de julio de 2020, http://bbc. in $/ 3 \mathrm{pFVwz} 0$.

26. Jeffrey Gogo, "Crypto Hardware Wallet Firm Ledger Hacked, One Million Customer Emails Exposed", News Bitcoin, 29 de julio de 2020, http://bit.ly/382Lkul.

27. Valeska Escobar, "Superfinanciero colombiano: denuncias por estafas con supuestas criptomonedas no cuentan con respaldo Estatal”, Criptonoticias, 20 de julio de 2017, http://bit.ly/3bVWKRo. 
donde ya existen regulaciones implementadas para encuadrar este fenómeno, ${ }^{28}$ de las cuales los países suramericanos podrían tomar ejemplo.

\section{La Regulación JURÍdica en COLOMBia}

La primera entidad en pronunciarse sobre el tema fue la Superintendencia Financiera, a través de la Carta Circular n. ${ }^{\circ} 29$ del 26 de marzo de 2014, quien teniendo en cuenta el auge de las "monedas virtuales", manifestó que dichos instrumentos no estaban respaldados ni regulados por ninguna autoridad monetaria, ni por un activo físico. De igual forma, la entidad resaltó que según la Ley 31 de 1992, el peso es el único medio de pago de curso legal en Colombia, por tanto, el bitcóin no puede ser considerado legalmente como una moneda.

Por otro lado, la Superfinanciera resaltó los riesgos a los cuales se encontraban expuestos los individuos que comerciaran con criptomonedas, en especial su alta volatilidad, su descentralización y la ausencia de respaldo en activos físicos. También indicó que ninguna de las plataformas de transacción de este tipo de criptoactivos, como el bitcóin, se encuentran reguladas por la legislación colombiana y, por tanto, no son objeto de control, vigilancia o inspección por parte de la entidad. Finalizó diciendo que las entidades bajo vigilancia de la Superintendencia, no se encontraban autorizadas para custodiar, invertir, ni intermediar con este tipo de instrumentos.

Dos años después, fue el Banco de la República quien emitió su concepto n. ${ }^{\circ} 20348$ de 2016, en el cual reiteró que la única moneda de curso legal en Colombia es el peso y se acogió a lo dicho anteriormente por la Superfinanciera.

Tributariamente, la Dirección de Impuestos y Aduana Nacionales (DIAN), en su concepto n. ${ }^{\circ} 20436$ de 2017, dando respuesta a una solicitud formulada ante la Cámara de Comercio de Bogotá, respecto a si ¿minar monedas virtuales constituye una actividad gravada en Colombia con el impuesto sobre la renta?, la entidad respondió afirmativamente.

De igual forma, precisó que la moneda virtual es considerada como:

Una representación digital de valor que puede ser comerciada digitalmente y funciona como (1) un medio de cambio; y/o (2) una unidad de cuenta; y/o (3) un depósito de valor, pero no tiene curso legal (es decir, cuando se ofrece a un acreedor, es una oferta válida y legal de pago) en ninguna jurisdicción.

28. Francia, "LOI PACTE $n .^{\circ} 2019-486$ du 22 mai 2019 relative à la croissance et la transformation des entreprises". 
La DIAN se acogió a los conceptos mencionados anteriormente por la Superfinanciera y por el Banco de la República y, posteriormente, hizo una explicación del sistema de minería con el que funciona bitcóin, determinando que dicha actividad al generar rendimientos gracias a la utilización de energía y de poder de computo, es objeto de tributación, conforme al artículo 9 del Estatuto Tributario.

Por último, a la fecha no hay ningún pronunciamiento oficial ni normatividad clara que estipule si las actividades de intercambio, compra y venta de criptomonedas o ganancias producto de trading deban ser declaradas $y$, por ende, objeto de impuestos. ${ }^{29}$

Así las cosas, hasta 2017 no existía una regulación definida sobre las criptomonedas, solamente conceptos de diferentes entidades que apuntaban a tener cuidado con su utilización. Sin embargo, al tratarse de aspectos tributarios, el Estado no desea dejar de percibir impuestos, pese a la ausencia de regulación.

Debido a esta falta de claridad y de armonización, el Congreso colombiano presentó el Proyecto de Ley n. ${ }^{\circ} 028$ de 2018, el cual pretende definir las monedas virtuales y regular sus operaciones en el territorio colombiano. Este define una criptomoneda como un: "Activo de carácter virtual, el cual representa un valor que se registra de forma electrónica y que puede ser utilizado por las personas como forma de pago para cualquier tipo de acto jurídico y cuya transferencia únicamente puede llevarse a cabo a través de medios electrónicos".

De igual forma, el proyecto pretende regular a los actores del ecosistema, como las plataformas de intercambio, así como el trading de criptomonedas, su tributación, impuestos y sanciones. Dicha ley se encuentra en curso y aún no ha sido aprobada y pese a que no prohíbe el uso de los criptoactivos, la carga impositiva de un $5 \%$, con la que pretenden gravar las operaciones en criptomonedas, así como las sanciones estipuladas, tienen el riesgo de desmotivar fuertemente la adopción y el progreso de esta tecnología en Colombia.

Según un estudio reciente, ${ }^{30}$ en el país se intercambian diariamente alrededor de $\$ 500.000$ en bitcóins, siendo uno de los más activos en Latinoamérica. Además, dicha cifra debe ser mucho más alta en la actualidad, teniendo en cuenta que la criptomoneda ha visto un aumento significativo en precio desde el mes de diciembre de 2020 , llegando a nuevos máximos históricos y alcanzando superar los $\$ 50.000$ en el mes de febrero de 2021.

29. “CCómo declarar sus Bitcoins a la hora de pagar impuestos?”, Colombia Fintech, 8 de septiembre de 2020, http://bit.ly/3n581SR.

30. "Colombia le apuesta a los Bitcoins", Colombia Fintech, 8 de septiembre de 2020, http://bit.ly/3n19r0D. 


\section{LA REgUlaCión JURídica en ECUAdOR}

En Ecuador, el panorama jurídico del bitcóin y en general de las criptomonedas no es más alentador que en Colombia. En el año 2014 estas fueron prohibidas a través de una reforma legislativa al Código Monetario y Financiero, autorizada por la Asamblea Nacional, ${ }^{31}$ quien apoyó el proyecto de Ley, el cual le otorgó al Gobierno la autorización para hacer pagos en dinero electrónico y monopolizar el control y fabricación de moneda virtual. El artículo 94 de la Ley, estipula: "El Banco Central del Ecuador es la única entidad autorizada para proveer y gestionar moneda metálica nacional o electrónica en la República del Ecuador, equivalente y convertible a dólares de los Estados Unidos de América". ${ }^{32}$

Poco después de su publicación, la Asamblea Nacional informó que el dinero electrónico traería beneficios para la economía del país, atrayendo a ciudadanos ecuatorianos, especialmente a los no bancarizados y que dicha moneda electrónica sería desarrollada por el gobierno y estaría respaldada por los activos del Banco Central.

En síntesis, el país desde el año 2014 mostró su hostilidad a la utilización de las criptomonedas descentralizadas por estar fuera de su control, por lo que prefirió adoptar la idea, pero desnaturalizándola y acomodándola en función de sus intereses, estableciendo un sistema de dinero electrónico ${ }^{33}$ muy diferente a las criptomonedas, que no tuvo mayor acogida entre la población.

Como era de esperarse, esta postura desalentó el progreso y la utilización de monedas digitales en el territorio, incluyendo bitcóin, ya que el artículo 98 de la Ley prohíbe de forma general: "La emisión, reproducción, imitación, falsificación o simulación total o parcial de moneda y dinero, así como su circulación por cualquier medio, soporte o forma de representación". ${ }^{34}$

Lo que significa que las plataformas de intercambio registradas en el país se ven afectadas por dicha medida. Las sanciones previstas son tan fuertes, que incluso las conductas contrarias a la ley son sancionadas penalmente y el decomiso de los bitcóins o demás criptoactivos están autorizados. ${ }^{35}$

31. Ecuador, Asamblea Nacional, Código Orgánico Monetario y Financiero, Registro Oficial n. ${ }^{\circ} 332$, segundo suplemento, 12 de septiembre de 2014.

32. Ibíd., artículo 94.

33. Susana Rojas, "El dinero electrónico en Ecuador, ¿qué es y cómo funciona?", GK City, 8 de septiembre de 2019, http://bit.ly/3csNioP.

34. Ibíd., artículo 98.

35. Angel Di Matteo, "Ecuador prohíbe el uso de Bitcoin y anuncia la creación de una nueva moneda digital", DiarioBitcoin, 25 de julio de 2014, https://bit.ly/380xuIM. 
En el año 2018, el Banco Central de Ecuador, confirmó la postura adoptada por el gobierno, manifestando en un comunicado presente en su sitio web, que "el uso de las criptomonedas o medios digitales que se utilizan para invertir y realizar transacciones a través de internet, no está autorizado en el país", concepto respaldado por la Junta de Política y Regulación Monetaria de Ecuador, quien reiteró que todas las actividades relacionadas con monedas virtuales se encuentran restringidas. ${ }^{36}$

En consecuencia, el ecosistema cripto en Ecuador no es uno de los más atractivos y esto impide de igual forma el desarrollo de otras iniciativas basadas en la tecnología Blockchain o en la minería de criptomonedas. Según un estudio realizado por periodistas de Diariobitcoin, pese al bajo costo de la electricidad comparado con otros países de Latinoamérica, la minería física no es popular en el país debido a las restricciones.

Las monedas más populares son bitcóin, Ripple, Ethereum, Eos, Dash y Litecoin y, además, existe una criptomoneda propia llamada Sucrecoin, la cual se focaliza en el manejo de remesas dentro y fuera del país; sin embargo, no hay mucha información al respecto, ni intercambios identificados en los cuales se pueda comerciar con ella.

Según el sitio web CoinMap, ${ }^{37}$ hay cierta actividad de comercios que aceptan criptomonedas en el territorio nacional, sobre todo en Quito, Guayaquil y Cuenca; no obstante, esta es mínima comparada con algunos otros países, incluyendo Colombia. En el caso de los cajeros automáticos de criptomonedas, el sitio web CoinATM Radar, indica que actualmente existen tres cajeros disponibles en Ecuador, dos en Quito y uno en Tena, los cuales, jurídicamente, estarían prestando un servicio ilegal.

Por último, en cuanto a las plataformas de intercambio, varias que operaban anteriormente han desaparecido del territorio; no obstante, aún se encuentran plataformas como Capitalika, quien según su sitio web afirma prestar servicios de compra y venta de criptomonedas en Ecuador, aunque su sede legal se ubica en Estonia. La mayoría de operaciones de compra y venta de criptomonedas en el país se realizan a través de plataformas $P 2 P$ como LocalBitcóins, la cual se encarga de conectar usuarios para que ellos intercambien directamente los criptoactivos.

Este cuadro jurídico adverso a las criptomonedas, permite que estafadores se aprovechen de la situación e implementen estafas para defraudar a las personas que intentan invertir en este tipo de activos. Además, las fuertes regulaciones impiden el crecimiento de una industria cada vez más importante, es por esto que tal vez en un

36. Arnaldo Ochoa, “Todo sobre Bitcoin y criptomonedas en Ecuador”, DiarioBitcoin, 11 de agosto de 2020, https://bit.ly/2KgjefD.

37. "All the cryptocurrency merchants and ATMs of the world in one map", CoinMap, accedido 30 de diciembre de 2020, http://bit.ly/3rGNbNd. 
futuro cercano sea posible la modificación de la ley para permitir la utilización de esta tecnología y garantizar un ambiente regulado y seguro para que el país y sus ciudadanos estén en sintonía con los avances monetarios de la era digital.

\section{CONCLUSIONES}

Las criptomonedas y en especial el bitcóin, ofrecen verdaderas funcionalidades y posibilidades que están revolucionando el sistema financiero tradicional y otros sectores de la sociedad. Su crecimiento progresivo y la adopción cada vez mayor por parte de individuos, instituciones privadas y gobiernos demuestra su aceptación y sus grandes proyecciones de crecimiento.

Colombia y Ecuador no demuestran estar a la vanguardia en este tema. En el caso colombiano, las medidas legislativas que se encuentran en curso en el Congreso podrían desmotivar fuertemente el desarrollo y adopción de esta tecnología y Ecuador, por su parte, no parece querer cambiar su legislación adversa al florecimiento de las criptomonedas. Sin embargo, es necesario que estos dos países adopten una regulación menos restrictiva que motive el florecimiento de la industria y la adopción de los criptoactivos, garantizando a su vez la protección de los individuos. Esto teniendo en cuenta los bajos costes de electricidad que tienen ambos países, así como el interés de sus habitantes en este tema.

Una regulación jurídica amigable con el ecosistema cripto, atraería directamente la inversión y el desarrollo de estos proyectos en el territorio, lo que directamente implicaría un crecimiento para la economía, no solo en el ámbito financiero, sino también en otros sectores que puedan verse beneficiados de tecnologías como la Blockchain y la minería.

Es tal vez por este motivo, que algunas luces exploratorias comienzan a surgir, principalmente encontramos el Sandbox regulatorio ${ }^{38}$ que pretende implementar la Superintendencia Financiera de Colombia con diferentes actores del sistema financiero tradicional y del ecosistema cripto, así como la reciente la Ley de Modernización a la Ley de Compañías de Ecuador del 1 de diciembre de 2020, que busca acercar e implementar servicios de criptografía y de Blockchain en las industrias ecuatorianas.

Finalmente, es importante que los gobiernos contribuyan con iniciativas sociales que impliquen la formación y educación en estas nuevas tecnologías. De esta forma, las criptomonedas serían más accesibles a todos, lo que directamente aumentaría la

38. Paola Vargas, "En el sandbox, habrá nueve alianzas que podrán realizar pruebas con criptoactivos", $L a$ República, 29 de enero de 2021, http://bit.ly/3dZO7rA. 
fortaleza y el crecimiento de su red. Así mismo, varias de las funcionalidades para las cuales el bitcóin fue creado y que, en síntesis, consisten en el reemplazo del dinero fiduciario por uno respaldado en algoritmos matemáticos y en la confianza de los propios usuarios que utilizan la red, podrían estar cada vez más cerca de la realidad.

\section{BIBLIOGRAFÍA}

Agencia EFE. "La inflación en Venezuela fue del 1433,58\% hasta septiembre, según el Parlamento", 8 de octubre de 2020. http://bit.ly/3htG8mm.

Ammous, Saifedean. El Patrón Bitcoin. La alternativa descentralizada a los bancos centrales. Barcelona: Deusto Grupo Planeta, 2018.

Aufray, Christophe. "Le canton Suisse de Zoug accepte Bitcoin et Ether pour les impôts". Cryptonaute, 7 de septiembre de 2020. http://bit.ly/3aWjGAZ.

Banco de la República de Colombia. "Comentarios sobre la regulación de monedas virtuales", 22 de septiembre de 2016. https://bit.ly/382nzT5.

Bayard, Florian. "Le Bitcoin (BTC) est-il trop dépendant de la Chine?". Cryptoast, 29 de septiembre de 2020. http://bit.ly/38NnqSE.

BBC News. Twitter hack: Exchange "blocked 1,000 Bitcoin transactions", 21 de julio de 2020. http://bbc.in/3pFVwz0.

Bit2Me Academy. “¿Qué es la criptografía?”. Accedido 30 de diciembre de 2020. https://bit. ly/382i2vT.

Blog Bankia. "Historia del dinero: del trueque a las criptomonedas", 26 de abril de 2016. http://bit.ly/2XbWy9L.

Caballero, Federico. "La inflación en Argentina: Una explicación monetaria". Economipedia Blog, 14 de septiembre de 2020. http://bit.ly/3aRthJo.

Champagne, Phil. El libro de Satoshi. Blockchain. España, 2014.

Colombia. Congreso de la República de Colombia. "Proyecto de Ley 028 de 2018 por el cual se regula el uso de monedas virtuales". Accedido 30 de diciembre de 2020. http://bit. ly/3nS4jfO.

Colombia Fintech. “¿Cómo declarar sus Bitcoins a la hora de pagar impuestos?”, 8 de septiembre de 2020. http://bit.ly/3n581SR.

Colombia Fintech. "Colombia le apuesta a los Bitcoins", 8 de septiembre de 2020. http://bit. ly/3n19r0D.

Comisión para el Mercado Financiero. "Historia de los bancos". CMFEduca. Accedido 30 de diciembre de 2020. https://bit.ly/3hAvom3.

Cripto 247. “¿Cuáles son los 6 países que más comerciaron con bitcoin en Latinoamérica?”, 16 de junio de 2020. https://bit.ly/394EVyb.

Di Matteo, Angel. "Ecuador prohíbe el uso de Bitcoin y anuncia la creación de una nueva moneda digital". DiarioBitcoin, 25 de julio de 2014. https://bit.ly/380xuIM. 
Dirección de Impuestos y Aduanas Nacionales-DIAN. Colombia. "Concepto 20436 del 2 de agosto de 2017-Minería de monedas virtuales está gravada con el impuesto de renta”. http://bit.ly/3aVW3bR.

Domat, Chloè. "Sa monnaie ne vaut plus rien, le Liban plonge dans l'abîme". Ouest France presse, 11 de julio de 2020. https://bit.ly/3n0z0z2.

Ecuador. Asamblea Nacional de Ecuador. Código Orgánico Monetario y Financiero de Ecuador (2020). Registro Oficial n. . 332, segundo suplemento, 12 de septiembre de 2014.

Francia. LOI PACTE n. ${ }^{\circ} 2019-486$ du 22 mai 2019 relative à la croissance et la transformation des entreprises.

Gogo, Jeffrey. “Crypto Hardware Wallet Firm Ledger Hacked, One Million Customer Emails Exposed". Bitcoin.com, 29 de julio de 2020. http://bit.ly/382Lkul.

INESE. "Pólizas de seguro para las criptomonedas, un nuevo mercado en auge", 28 de julio de 2020. http://bit.ly/2L3Fks9.

ING. "Cryptocurrencies and tokens". Accedido 25 de diciembre de 2020. https://bit. ly/2X0k8WQ.

Kapersky. “¿Qué es el cifrado de datos?”, 28 de noviembre de 2018. http://bit.ly/380cm5m.

Kiyosaki, Robert. FALSO: Dinero falso, falsos maestros, falsos activos: cómo las mentiras vuelven a los pobres y a la clase media más pobres. 2019.

Leal, Andrea. "2021 promete mayor expansión de granjas mineras de Bitcoin en Estados Unidos y Canadá". CriptoNoticias, 24 de diciembre de 2020. http://bit.ly/394K2OT.

Macrotrends.net. "U.S. Dollar Index.43 Year Historical Chart”. Accedido 30 de diciembre de 2020. http://bit.ly/2L6uUIq.

McFarland, Charles, Tim Hux, Eric Wuehler y Sean Campbell. "Informe sobre amenazas contra Blockchain". McAfee. Accedido 30 de diciembre de 2020. https://bit.ly/2JyVHN3.

Mordi, Eseandre. "Bitcoin Is Twice As Profitable As Gold And the S\&P500 Index In 2020". TheTradable, 1 de octubre de 2020. http://bit.ly/3b16BX5.

Nakamoto Satoshi. Bitcoin: Un sistema de pago electrónico persona a persona, 2009. Accedido 25 de diciembre de 2020. http://bit.ly/2WXNNQv.

Ochoa, Arnaldo. "Todo sobre Bitcoin y criptomonedas en Ecuador". DiarioBitcoin, 11 de agosto de 2020. https://bit.ly/2KGjefD.

Pastorino, Cecilia. “Blockchain: Qué es, cómo funciona y cómo se está usando en el mercado". WeLiveSecurity by Eset, 4 de septiembre de 2018. http://bit.ly/3pBCJo9.

Superintendencia Financiera de Colombia. Carta circular $n .^{\circ} 29$ del 26 de marzo de 2014, Riesgos de las operaciones realizadas con "Monedas virtuales". 